\title{
A computerised tailored intervention for increasing intakes of fruit, vegetables, brown bread and wholegrain cereals in adolescent girls
}

\author{
Gail Rees ${ }^{1, *}$, Savita Bakhshi ${ }^{2}$, Alecia Surujlal-Harry ${ }^{2}$, Mikis Stasinopoulos ${ }^{3}$ and \\ Anna Baker ${ }^{2}$ \\ ${ }^{1}$ School of Biological Sciences, University of Plymouth, Drake Circus, Plymouth PL4 8AA, UK: ${ }^{2}$ Department of \\ Psychology, London Metropolitan University, London, UK: ${ }^{3}$ STORM, London Metropolitan University, London, UK
}

Submitted 25 July 2008: Accepted 11 November 2009: First published online 11 January 2010

\begin{abstract}
Objective: To evaluate the effectiveness of a computer-generated tailored intervention leaflet compared with a generic leaflet aimed at increasing brown bread, wholegrain cereal, fruit and vegetable intakes in adolescent girls.

Design: Clustered randomised controlled trial. Dietary intake was assessed via three $24 \mathrm{~h}$ dietary recalls.

Setting: Eight secondary schools in areas of low income and/or high ethnic diversity, five in London and three in the West Midlands, UK.

Subjects: Girls aged 12-16 years participated ( $n$ 823) and were randomised by school class to receive either the tailored intervention ( $n$ 406) or a generic leaflet ( $n$ 417).

Results: At follow-up 637 (77\%) participants completed both baseline and follow-up dietary recalls. The tailored intervention leaflet had a statistically significant effect on brown bread intake (increasing from 0.39 to 0.51 servings/d) with a smaller but significant increase in the control group also (increasing from $0 \cdot 28$ to 0.35 servings/ d). The intervention group achieved 0.05 more servings of brown bread daily than the control group $(P<0 \cdot 05)$, which is equivalent to 0.35 servings/week. For the other foods there were no significant effects of the tailored intervention.

Conclusions: The intervention group consumed approximately 0.35 more servings of brown bread weekly than the control group from baseline. Although this change between groups was statistically significant the magnitude was small. Evaluation of the intervention was disappointing but the tailored leaflet was received more positively in some respects than the control leaflet. More needs to be done to increase motivation to change dietary intake in adolescent girls.
\end{abstract}

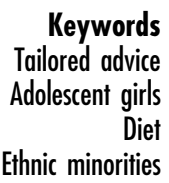

It is well recognised that improving the eating habits and nutritional status of young women is important. Not only do women have a major influence on the eating habits of their family, but during pregnancy the nutritional status of the mother will affect the health of her unborn child ${ }^{(1,2)}$. Poor nutritional status of the mother has been associated with low birth weight, which increases the risk of infant mortality and morbidity ${ }^{(3)}$.

In the UK micronutrient intakes are of concern in many teenagers, which is partly due to their low intakes of fruit, vegetables and wholegrain products. The National Diet and Nutrition Survey (NDNS) of young people ${ }^{(4)}$ found that girls aged 15-18 years consumed on average 2.5 portions of fruit and vegetables daily, equivalent to $50 \%$ of the recommended 5 portions/d, and one in five children ate no fruit at all in an average week. Those from less advantaged households on average consumed approxi- mately one less serving daily of fruit and vegetables combined. The NDNS ${ }^{(4)}$ also found that $90 \%$ of girls aged 11-18 years consumed white bread daily but consumption of high-fibre, wholegrain cereal and wholemeal bread contributed only $4-8 \%$ of total fibre intake.

It is thought that dietary changes need to occur preconception or very early in pregnancy to have maximum impact on birth weight ${ }^{(5)}$. Therefore, targeting nutritional interventions at teenage girls before they become pregnant is extremely important. Girls from low-income and some ethnic minority groups are more likely to have a teenage pregnancy ${ }^{(6)}$ and are more likely to have a lowbirth-weight baby ${ }^{(7)}$. Thus in these groups the need for nutritional interventions may be greater and necessary at an earlier stage.

Research has demonstrated that tailored interventions can be successful in changing dietary behaviour in adult 
populations $^{(8)}$ and have favourable responses in terms of acceptability $^{(9)}$. Tailoring of interventions can be done using a variety of factors such as demographic characteristics, psychological variables or psychological models. The nature of these interventions means that there is less redundant information and that recipients can focus on aspects that are more pertinent to them based on prior assessment $^{(10,11)}$. Furthermore, information delivered in this way is more likely to be read and remembered by participants, and also seems to have a greater impact on motivating people to change their eating behaviour compared with other forms of intervention ${ }^{(12,13)}$. Unlike individual counselling, tailored interventions can reach a large sample population especially when used over the Internet but still give individual feedback. A systematic review of randomised trials on the effectiveness of computer-tailored education on dietary behaviours found that twenty out of a total of twenty-six nutrition studies had a significant effect $^{(14)}$. However, there is a paucity of research using tailored interventions in adolescent samples and from ethnic minorities, particularly in the UK.

The aim of the present study was to evaluate the effectiveness of a computer-generated tailored intervention leaflet compared with a generic leaflet aimed at increasing brown bread, wholegrain cereal, fruit and vegetable intakes in adolescent girls. The intervention was tailored to a participant's self-reported intake of the target foods, and used psychological constructs from the Theory of Planned Behaviour $^{(15)}$ and the Transtheoretical Model ${ }^{(16)}$.

\section{Methods}

\section{Design}

The study was a randomised controlled cluster trial to evaluate the efficacy of a tailored intervention on increasing dietary intake of targeted foods in adolescent girls aged 12-16 years. The intervention group received a leaflet, tailored to their responses to a baseline diet and psychological questionnaire. All girls in the control group received a copy of a comparable generic leaflet based on national guidelines ${ }^{(17)}$, which was not tailored. Participants were assessed at baseline and then at followup three months later. The key outcomes were recallreported intakes of brown bread, wholegrain cereal, fruit and vegetables. Psychological outcomes were behavioural beliefs (cognitive and affective), normative beliefs and control beliefs about foods, and readiness to eat the target foods. These will be reported elsewhere.

The study was approved by the Department of Psychology Research Ethics Committee, London Metropolitan University and the Local NHS Research Ethics Committee. Parents were informed of the study by letter and could request for their child not to take part. All children were informed verbally and in writing about the study and gave written consent to participate.

\section{Study population}

Schools in two areas of the UK, London and the West Midlands, were contacted based on their representation of ethnic minority and low-income groups. Eleven schools out of sixty-nine (16\%) agreed to participate in the study. It was not possible to document the reasons why schools did not wish to participate as many schools just did not respond to the invitation or gave no reason. In total eight schools participated in the full study. Five of these were in London, three were in the West Midlands, four were girls-only and four were mixed schools. Boys were given a control leaflet and their data are not included in the present analysis. Students from five year groups (aged 12-16 years) participated. The number of classes included from each year group varied across the schools, subject to student availability.

A researcher who was not involved in the project randomly allocated classes of children to either the tailored intervention or control group. This was done for each school after completion of the baseline psychological questionnaire. Randomisation was done at the class level to ensure that intervention and control groups had an even spread of ethnicities and social groups. This would not have been possible if the school had been the unit of randomisation.

\section{Outcome and process measures}

A baseline psychological questionnaire consisting of thirty items for each food was developed from a preliminary questionnaire and focus group interviews that explored behavioural beliefs around the target foods and dietary change in the target population ${ }^{(18)}$. The final questionnaire was used to rate psychological factors from the Theory of Planned Behaviour ${ }^{(15)}$. Participants were asked to answer questions on behavioural beliefs (attitudes, and cognitive and affective beliefs), normative beliefs (subjective and descriptive norms), control beliefs (perceived behavioural control) and intention, which were analysed to create composite measures (Cronbach's $\alpha=0 \cdot 78-0 \cdot 88$ ) where appropriate. Additional questions on self-efficacy and stage of change were added. Self-efficacy was measured using one item: 'I am confident that I can eat ... every day'. Stage of change was assessed by a set of algorithms validated on other health behaviours and suitable for this population ${ }^{(16)}$. The intervention was tailored to these responses.

Participants were provided with three $24 \mathrm{~h}$ dietary recall sheets over three different days at baseline, and again at follow-up three months later, to record food and drink intake from the previous day. An example of a completed dietary sheet was provided as part of the pack so that participants could see how to record their food correctly. Recalls were completed during a class with help from the researchers. A timeline of the baseline and follow-up assessments can be seen in Fig. 1.

The numbers of portions of fruit and vegetables on the $24 \mathrm{~h}$ recall sheets were counted by a nutritionist following 


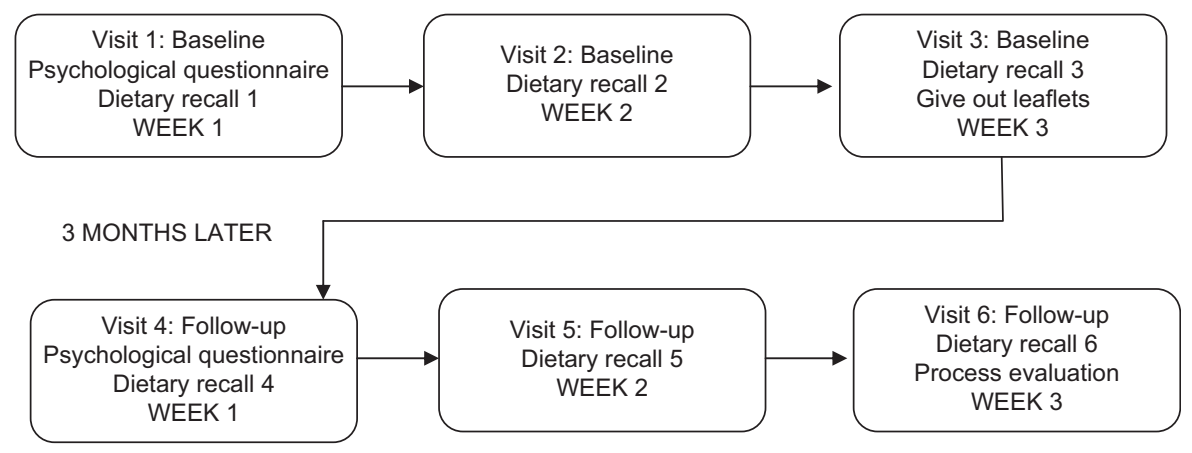

Fig. 1 Timeline for the baseline and follow-up assessments

the national guidelines ${ }^{(17)}$ and recalculated to produce a daily number. Where one dietary recall was missing, daily intake was calculated from two dietary recalls (Fig. 1). A random sample was double-checked for accuracy by a second nutritionist at baseline and follow-up with an inter-rater agreement of $96 \%$.

As there is no government recommendation on the frequency or portion size of whole grains, for the purposes of the current analysis one bowlful of wholegrain breakfast cereal and one slice of bread were taken to represent one portion. All high-fibre cereals, wholegrain cereals, muesli and oats counted as 'wholegrain'. Brown, granary and wholemeal breads were all counted as 'brown', again due to the difficulty in participants distinguishing them.

Participants were also asked to rate their consumption of target foods using a frequency measure ${ }^{(19)}$. These selfreported measures were used in the intervention leaflet to provide personalised feedback on current perceived intake but not as a main outcome for analysis. The more reliable measure of intake from the dietary recall could not be used in the personalisation of the intervention leaflets due to the short turnaround time in the production of the leaflets. Correlation coefficients between the frequency measure and recall-reported intake were $0 \cdot 27$ for brown bread, 0.32 for wholegrain cereal, 0.31 for vegetables and 0.45 for fruit (all $P<0 \cdot 001$ ).

Approximately 230 statements were developed to allow tailored responses to an individual's questionnaire data by a team of nutritionists and health psychologists based on qualitative interviews with adolescents. These statements were uploaded to a computer program so that leaflets could be automatically produced according to the questionnaire responses. A standard template for the leaflet was produced using colour graphics which was extensively piloted first with small groups of teenage girls. This allowed the leaflet to be adjusted to enhance visual appeal and relevance to the sample following feedback. The leaflets and process were then piloted with 248 girls from one school to ensure the leaflet was well received and the process workable.

A follow-up questionnaire assessed the same constructs as at baseline using an identical format to the baseline psychological questionnaire (results to be presented elsewhere) and three further dietary recalls were collected (Fig. 1).

An evaluation questionnaire was also administered that assessed the leaflet's comprehension, usefulness, content, meeting expectations, usability at present and in the future, personal relevance and information sharing with family. Five-point Likert-style responses were used ('strongly disagree' to 'strongly agree'). Data were categorised into positive ('agree' or 'strongly agree' with statement) or non-positive ('not sure', 'disagree' or 'strongly disagree') for perceptions of the leaflet.

\section{Sample size}

Sample size calculations were determined using GPower software version 2 (Department of Psychology, Heinrich Hein University, Dusseldorf, Germany) and published guidelines on effect sizes ${ }^{(20)}$. Revisions to the calculations were carried out based on dietary intake and class size information obtained from a pilot study. Estimates were based on three relevant statistical factors: (i) repeatedmeasures analysis for changes in behaviour between groups; (ii) intra-class correlations on dietary intake; and (iii) regression analysis of predictors of dietary change.

A total sample size of 200 was needed for repeatedmeasures analysis with an effect size of $F^{2}=0 \cdot 1, \alpha=0 \cdot 05$ and power of $98 \%$. Power was sufficiently high to reduce the incidence of Type 1 errors in data interpretation in all studies.

In addition to this calculation, possible intra-class effects needed to be taken into account. Using the intra-cluster correlation of each dietary behaviour from a preliminary study a new sample size was formulated as [initial sample size $(100)] \times 1+(m-1) p$, where $m=$ cluster size and $p=$ coefficient. Sample size was calculated according to an average cluster size of $m=15$ (class size) $^{\text {(21) }}$. A final sample size of 170 in each group was needed to detect small to medium changes in behaviour.

\section{Statistical analysis}

Analysis was conducted to examine whether there was a difference in the responses between the tailored intervention and control intervention groups. The impact of 
ethnicity, geographic location, school and age was also examined to take into account any clustering effects. Missing observations were treated as missing at random since it was considered that absence from school was not due to the effect of the intervention.

Three different statistical approaches were used to analyse the data: (i) the repeated measurement procedure in the SPSS statistical software package version 15 (SPSS Inc., Chicago, IL, USA); (ii) the random effect function lme of the package nlme in the R programming language (R Foundation for Statistical Computing, Vienna, Austria); and (iii) the experimental function gamllsNP in $\mathrm{R}$.

The lme function of the package nlme in $\mathrm{R}^{(22)}$ allowed the multilevel structure of the data to be taken into the account at the class, school and regional level. (Since there were only two regions this factor was entered as a fixed-effect term only and was found to be of no statistical significance.) The school/class/individuals relationship was first entered in the analysis as a multilevel (nested) random effect. The multilevel nesting structure was then tested by comparing the model 'school/class/individuals' to models with random-effect terms as (i) 'school/individuals', (ii) 'class/individuals' and (iii) 'individuals'. The simpler model (iii) with only individuals as a random effect proved to be statistically sufficient for the data. Both the SPSS and $\mathrm{R}$ analyses require a normality assumption for the response variable; therefore to test the robustness of the SPSS and $\mathrm{R}$ results a different approach using GAMLSS was tested, where a non-normal distribution was assumed for the response variable ${ }^{(23)}$. As the three methods produced no conflicting results, SPSS was used.

\section{Results}

\section{Recruitment of participants}

Out of a total of 830 girls who were invited to take part in the study, five girls declined and two parents declined on their child's behalf $(0 \cdot 8 \%)$. Therefore 823 participants were recruited at baseline after completion of the baseline psychological questionnaire, with 406 randomised to the tailored intervention group and 417 to the control group. Of the 823 who participated, 757 completed two or three dietary recalls and therefore were able to be evaluated at follow-up on dietary intake. Those who completed fewer than two out of three dietary recalls also received the intervention but were assessed on psychological factors only. At follow-up 637 participants completed both baseline and follow-up dietary recalls and the follow-up psychological questionnaire ( $84 \%$ of 757 ), with $323(51 \%)$ in the tailored intervention group and $314(49 \%)$ in the control group ( $84 \%$; Fig. 2). Overall there was a good retention rate with only two out of 823 girls withdrawing from the study. Dietary data were available for $92 \%$ of those recruited at baseline, with an attrition rate of $16 \%$ for completion of follow-up dietary recalls (due to absence from school). Those who did not complete all recalls did not differ in demographic characteristics or exposure to the intervention.

Participants were aged between 12 and 16 years with the majority being white $(53 \cdot 4 \%) ; 18.7 \%$ described themselves as Asian, 15.8\% black, 10.0\% mixed race and $2 \cdot 1 \%$ another ethnicity. The sample came from London (73.6\%) and the West Midlands (26.4\%).

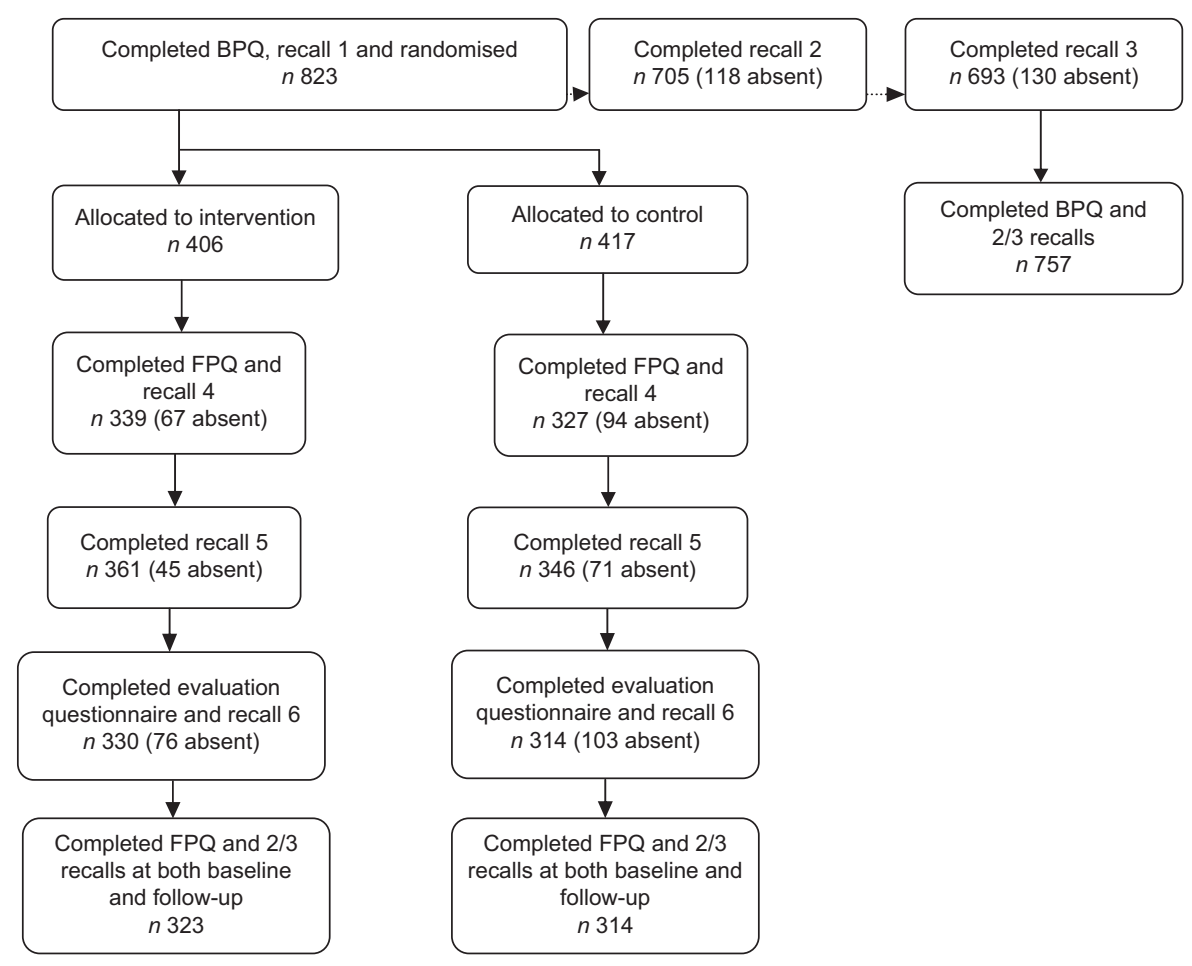

Fig. 2 Recruitment and participation flowchart (BPQ, baseline psychological questionnaire; FPQ, follow-up psychological questionnaire) 
Table 1 Daily dietary intake (number of servings/d) at baseline $(n 757)$ and percentage achieving target level*: adolescent girls, aged $12-16$ years, from secondary schools in areas of low income and/or high ethnic diversity, London and West Midlands, UK

\begin{tabular}{|c|c|c|c|c|c|c|c|c|}
\hline & \multicolumn{4}{|c|}{ Intervention } & \multicolumn{4}{|c|}{ Control } \\
\hline & Mean & SD & Range & $\%$ achieving target level & Mean & SD & Range & $\%$ achieving target level \\
\hline Brown bread & 0.39 & 0.74 & $0-4 \cdot 67$ & $17 \cdot 0$ & 0.28 & 0.58 & $0-3.33$ & $14 \cdot 0$ \\
\hline Wholegrain cereal & $0 \cdot 17$ & 0.31 & $0-1.50$ & $6 \cdot 6$ & $0 \cdot 15$ & 0.29 & $0-1 \cdot 50$ & $5 \cdot 0$ \\
\hline Fruit & 0.99 & 1.00 & $0-7 \cdot 25$ & $2 \cdot 7$ & 0.95 & 0.98 & $0-7 \cdot 25$ & $1 \cdot 8$ \\
\hline Vegetables & 0.69 & 0.60 & $0-3 \cdot 00$ & & 0.68 & 0.64 & $0-4 \cdot 33$ & \\
\hline
\end{tabular}

Girls in the intervention group subsequently received a computer-generated tailored intervention leaflet while girls in the control group received a generic leaflet aimed at increasing brown bread, wholegrain cereal, fruit and vegetable intakes.

${ }^{*}$ Target level for fruit and vegetables is at least 5 servings daily based on government advice. As no government target or recommended portion size exists for wholegrain bread and cereals, a target of one serving daily was set. This was equivalent to one slice of bread or one bowl of cereal.

\section{Dietary intake at baseline}

Dietary intake at baseline for brown bread, wholegrain cereal, fruit and vegetables showed low levels of consumption for all foods (Table 1 ). The average daily intake of brown bread was 0.34 servings equivalent to one slice of brown bread every $3 \mathrm{~d}$, while wholegrain cereal was $0 \cdot 16$ servings daily equivalent to one bowl of wholegrain cereal weekly. Daily fruit and vegetables consumption combined was 1.66 (SD 1.27) servings daily. Very few girls consumed the recommended 5 servings of fruit and vegetables daily (2.5\%; Table 1$)$, less than $16 \%$ consumed brown bread daily and less than $6 \%$ ate wholegrain cereal daily.

\section{Changes to dietary intake}

The tailored intervention leaflet had a statistically significant effect on brown bread intake compared with the control leaflet (Table 2). However, there were no school, location, year or ethnicity effects in the model. This indicates that this particular dietary behaviour changed in the intervention group with no contribution from demographic characteristics. Intake of brown bread increased from 0.39 to 0.51 servings/d in the intervention group, with a smaller but significant increase in the control group also (from $0 \cdot 28$ to $0 \cdot 35$ servings/d; Table 2 ).

For the other foods there were no significant effects of the tailored intervention above that of the control intervention. There was no statistically significant effect of intervention, school, year or ethnicity in the model for wholegrain cereal. However there was a location effect, whereby those participants from the West Midlands were more likely to decrease their intake regardless of intervention.

For fruit intake there was a significant location effect but no intervention, school, year or ethnicity effect in the model. Therefore participants from West Midlands were more likely to make changes to their intake regardless of intervention. Results showed a significant decrease in fruit intake for both intervention and control groups by approximately one serving per week.

There was also a strong ethnicity and location effect for vegetables, showing similar findings to those for wholegrain cereal. However there was also a weak statistical effect of school year but no intervention or school effect.
Table 2 Change in dietary intake (number of servings/d) from baseline to follow-up: adolescent girls, aged 12-16 years, from secondary schools in areas of low income and/or high ethnic diversity, London and West Midlands, UK

\begin{tabular}{lcc}
\hline & Intervention & Control \\
\hline Brown bread & $0.12^{\mathrm{a}, \mathrm{b}}$ & $0.07^{\mathrm{a}}$ \\
Wholegrain cereal & 0.00 & -0.03 \\
Fruit & $-0.15^{\mathrm{a}}$ & $-0.11^{\mathrm{a}}$ \\
Vegetables & 0.00 & -0.03 \\
\hline
\end{tabular}

Girls in the intervention group received a computer-generated tailored intervention leaflet while girls in the control group received a generic leaflet aimed at increasing brown bread, wholegrain cereal, fruit and vegetable intakes. Significant effect of ${ }^{a}$ time and ${ }^{b}$ interaction of time $\times$ condition $(P<0.05)$, controlling for school, location, school year and ethnicity.

Those from London were more likely to increase intake of vegetables. There were also some effects of ethnicity but only in those groups with small sample sizes. Overall there was no change to vegetable intake.

\section{Evaluation of the intervention}

There were significant differences in feedback about the leaflet (Table 3). More participants in the tailored group perceived the leaflet easy to use, easy to understand and personally relevant compared with the control group. Although the numbers of participants who reported using the information to make changes to their diet (28\% tailored, $22 \%$ control) and sharing the information with their family (27\% tailored, 14\% control) were disappointingly low, the rates were higher in the tailored group.

Disappointingly, less than a third of girls in both the intervention and control groups reported using their leaflets to make changes to their diet, and further, less than a fifth still referred to the leaflets three months after the intervention was received.

\section{Discussion}

Dietary assessment at baseline showed that the majority of the sample $(97 \cdot 5 \%)$ did not eat 5 servings of fruit and vegetables daily, which is a far higher percentage not meeting recommended levels than in the general population $^{(4)}$. This may be due to the inclusion of different 
Table 3 Perception of leaflets in the intervention and control groups (positive scores): adolescent girls, aged 12-16 years, from secondary schools in areas of low income and/or high ethnic diversity, London and West Midlands, UK

\begin{tabular}{|c|c|c|c|}
\hline & Intervention & Control & $x^{2}$ \\
\hline The leaflet was easy to use (\%) & 64 & 52 & $8 \cdot 4^{\star \star}$ \\
\hline The leaflet was easy to understand (\%) & 69 & 61 & $4 \cdot 11^{*}$ \\
\hline The leaflet contained the right amount of information (\%) & 38 & 37 & $0 \cdot 10$ \\
\hline The information was personally relevant to me (\%) & 34 & 18 & $18 \cdot 3^{\star \star \star}$ \\
\hline I have used the information provided to make changes to my diet (\%) & 28 & 22 & $3 \cdot 13$ \\
\hline I still refer to the leaflet for healthy eating advice (\%) & 17 & 19 & $0 \cdot 27$ \\
\hline I shared the information in the leaflet with my family (\%) & 27 & 14 & $13 \cdot 4^{\star \star *}$ \\
\hline
\end{tabular}

Girls in the intervention group received a computer-generated tailored intervention leaflet while girls in the control group received a generic leaflet aimed at increasing brown bread, wholegrain cereal, fruit and vegetable intakes.

Significant difference between groups: ${ }^{\star} P<0.05,{ }^{\star \star} P<0.01,{ }^{* \star \star} P<0.001$.

ethnic groups in our sample and/or the fact it was a lower than average income sample. Also, the dietary recall method used in our study is more likely to produce under-reporting of dietary intakes compared with the $7 \mathrm{~d}$ weighed intake method used by the NDNS ${ }^{(24)}$. The intake of brown bread and wholegrain cereal was also extremely low, with the average intake approximately 0.3 servings/ $\mathrm{d}$ for brown bread and $0 \cdot 2$ servings/d for wholegrain cereal. Only $16 \%$ of girls consumed brown bread daily and $8 \%$ consumed wholegrain cereal daily.

Although both the intervention and control groups showed a significant increase in brown bread intake, the tailored leaflet had a statistically significant effect over the control leaflet. However, the magnitude of this was small $(+0.12$ servings/d in intervention group compared with +0.07 servings/ $\mathrm{d}$ in control group). There were no changes at all for either wholegrain cereal or vegetables, where intake remained stable between baseline and follow-up. Intake for fruit decreased between baseline and follow-up for both the intervention group and the control group by approximately one serving per week. Data collection was completed outside those months which are subject to seasonal variation, so the negative changes to fruit intake must be explained by other factors. One explanation may be that while consumption of brown bread increased, the effect on overall eating patterns was a decrease in fruit. Perhaps girls were consuming brown bread instead of fruit (e.g. snacks after school) or they felt fuller as consequence of consuming more complex carbohydrates. However, the changes to brown bread and fruit were very small, so it seems unlikely that this would be the case.

In contrast to our study, the majority of tailored interventions on adults have shown positive increases in intake of fruit and vegetables ${ }^{(8,10,25,26)}$. Giroti et al. ${ }^{(27)}$ found changes for some of their non-adult participants when information was tailored to academic level but only in older students. There is a lack of research specifically on adolescents, where interventions have been tailored to psychological or theoretical factors, with which to compare our findings. However, research with younger children has shown similar results in a school setting. Mangunkusumo et $a l .{ }^{(28)}$ were unable to demonstrate changes in intake using tailoring with brief counselling.
Using environmental changes as well as tailored computer feedback Haerens et al. $^{(29)}$ saw a reduction in fat intake in girls but produced no change in fruit intake.

There is some similarity in our findings to studies which have focused on more than one food type or more than one behaviour, where there is a tendency for change in some but not all behaviours or foods ${ }^{(27,30)}$. Both Campbell et $a l^{(31)}$ and Brug et $a l^{(8)}$ found a reduction in fat intake but no change in intake of fruit or vegetables in their tailored intervention. Campbell et al. found a decrease of about 10\% in combined fruit and vegetable intake at follow-up. It may be that participants prioritise what they want to change what is most relevant for them - and are not ready to make a complete dietary overhaul. We cannot determine whether the brown bread section was more relevant or alternatively, because the section on brown bread was first, they decided not to read the remainder of the leaflet.

There is a general lack of comparable research on interventions targeting complex carbohydrates. One intervention study ${ }^{(32)}$ aimed at increasing complex carbohydrate intake in families was successful, but fruit and vegetable intake was reduced in response. This finding highlights the difficulty of changing different components of the diet without associated and perhaps negative effects on non-targeted components. Careful consideration of the type of information given needs to be made, to focus on how foods fit into daily patterns rather than on specific foods out of context.

Evaluation of the tailored intervention showed disappointingly low numbers of girls (28\%) reporting using the leaflet to make changes to their diet (Table 3). However, the tailored leaflet had some positive benefits over the control leaflet, such as being easier to use and to understand, and being more personally relevant. Twice as many girls who received the tailored leaflet also referred to sharing their information with their family than in the control group, although the numbers were still low (27\% v. $14 \%)$.

\section{Limitations}

The order of presentation of information in the leaflets was not varied and it is possible that girls only responded to information at the start, which referred to brown bread. 
It is also possible that there was too much information included in the leaflet, as the evaluation data showed that only $38 \%$ thought the leaflet contained the right amount of information. The complexity and amount of information should be reduced in future. Incorporating wholegrain cereal and brown bread in addition to fruit and vegetables made the leaflet more complex to produce and use. One of the criticisms from schools about the project was that the intervention was available at only one time point. The schools were keen to have a healthy eating resource which could be more interactive. This would enable greater reinforcement of positive messages to encourage behavioural changes and also engagement with programmes. This is especially important for children from low-income groups for whom school may be the only source of health promotion messages. These findings are useful in informing future practice using tailored intervention leaflets in this sample.

The fact that only $34 \%$ of the tailored intervention sample thought the information was personally relevant to them is surprising, as a great deal of effort was directed during piloting to ensure messages were regarded as relevant to the sample. However, this may be because of the time delay of 3 months between administering the intervention and assessing impact. While the intervention may have had some success at the time which may be partially sustained, recall of the information over time is likely to be limited and thus the apparent personal impact reduced.

Dietary recall was collected for the majority of students on a school day. Ideally one of the dietary recalls should have been performed on a Monday in order to reflect intake on the preceding Sunday. However, this was not always possible to arrange with schools. Therefore, the data could be an under-representation of general intake as most girls reported that they consumed more of the target foods at the weekends.

The amount of detail given by the participants in the dietary recall was limited. For example, the most detail that girls could describe for bread was whether it was brown or white and the number of slices. We therefore could not detect if slices were thick or thin, or whether the bread was brown or wholemeal. For breakfast cereal participants reported their consumption by the bowlful. We did not try to distinguish between large or small bowls as our intention was to keep the process as simple as possible considering the age of the participants.

It could therefore be argued that the dietary assessment was not an accurate enough reflection of intake. However, we would have been able to detect a change from white to brown bread or an increase in the number of slices of brown bread. Likewise, while we could not detect a change if participants simply increased the amount of cereal in their bowl, we would have been able to detect a change from a low- to high-fibre cereal or an increase in the number of bowlfuls eaten.
Unlike many intervention studies, the current study included all participants at schools. Although this avoided the self-selection bias associated with many interventions, many girls clearly had no interest in changing their diet. This disinterest could explain the lack of success of our intervention compared with other tailored intervention studies which have used more motivated clients.

Motivation in an adolescent sample could possibly be improved by using an interactive online method. Internetbased interventions have shown good results in comparison to paper-based interventions ${ }^{(25)}$. Recent research indicates that the Internet is a useful method not only for collecting dietary and psychological data ${ }^{(33,34)}$ but also for addressing health behaviours in adolescents with equivalent acceptability to paper-based leaflets ${ }^{(35,36)}$. The benefits of using Internet-based interventions include convenience, novelty and appearance as well as flexibility of use in terms of automated data collection and openness of communication ${ }^{(37)}$. Having automatic generation of the tailored intervention means the intervention can be delivered immediately rather than waiting for data entry and printing. Additionally, it can cut down the time in completing the baseline assessment measures and enable data to be collected on different days. It may also be important in evaluating the efficacy of interventions to follow up participants using more qualitative methods, to look at differences between those who made change and those who did not. Further to this it is important to establish the relationship between changes in psychological factors and intake to understand the processes by which adolescents may change their behaviour.

\section{Conclusion}

The current intervention showed a small change in one dietary behaviour. Future work should focus on increasing motivation to change in this population and involve adolescents fully in the design and development of such programmes to improve usability and user engagement.

\section{Acknowledgements}

The study was commissioned by the Food Standards Agency (project number N14002). Work was carried out at London Metropolitan University, London, UK. The authors confirm that there is no conflict of interest. Each author has approved and contributed to the final manuscript. G.R. and A.B. generated the original research hypothesis, designed the study, supervised the research and wrote the manuscript. S.B. and A.S.-H. carried out the fieldwork, collated data, maintained the database and produced the intervention. M.S. contributed to the study design and conducted the statistical analysis. We would like to thank the children and schools for participating. 


\section{References}

1. Cox DN \& Anderson AS (2004) Food choice. In Public Health Nutrition, p. 152 [MJ Gibney, BM Margetts, JM Kearney et al., editors]. Oxford: Blackwell Publishing.

2. Kramer MS (1987) Determinants of low birth weight: methodological assessment and meta-analysis. Bull World Health Organ 65, 663-737.

3. Scottish Low Birthweight Study Group (1992) The Scottish Low Birthweight Study: I Survival. Growth, neuromotor and sensory impairment. Arch Dis Child 67, 675-681.

4. Gregory J, Lowe S, Bates C et al. (2000) National Diet and Nutrition Survey: Young People Aged 4 to 18 years. London: The Stationery Office.

5. Doyle W, Crawford MA, Wynn AHA et al. (1990) The association between maternal diet and birth dimensions. J Nutr Med 1, 9-17.

6. Health Education Authority (1999) Promoting the Health of Teenage and Lone Mothers. Setting a Research Agenda. Report of the Health Education Authority Expert Working Group chaired by Kaye Wellings. London: Health Education Authority.

7. Department of Health (2005) NHS Maternity Statistics, England: 2003-2004. London: Department of Health.

8. Brug J, Steenhuis I, Van Assema P et al. (1996) The impact of a computer-tailored nutrition intervention. Prev Med $\mathbf{2 5}$ 236-242.

9. Baker AH \& Wardle J (2002) Increasing fruit and vegetable intake among adults attending colorectal cancer screening: the efficacy of a brief tailored intervention. Cancer Epidemiol Biomarkers Prev 11, 203-206.

10. Baker AH \& Wardle J (1999) Low intensity, high impact: can low intensity interventions change behaviour? Presented at Psychology and the Renaissance of Health, 13th Conference of the European Health Psychology Society, Florence, Italy, 1-3 October 1999.

11. De Vries H \& Brug J (1999) Computer-tailored interventions motivating people to adopt health promoting behaviours: introduction to a new approach. Patient Educ Couns 36, 99-105.

12. Ryan P \& Lauver DR (2002) The efficacy of tailored interventions. J Nursing Scholarsh 4, 331-337.

13. Brug J, Campbell M \& van Assema P (1999) The application and impact of computer-generated personalized nutrition education: a review of the literature. Patient Educ Couns 36, 145-156.

14. Kroeze W, Werkman A \& Brug J (2006) A systematic review of randomized trials on the effectiveness of computertailored education on physical activity and dietary behaviors. Ann Behav Med 31, 205-223.

15. Ajzen I (1991) The theory of planned behaviour. Organ Behav Hum Decis Process 50, 179-211.

16. Cardinal B (1995) Methods, issues, and results in evaluation and research: construct validity of stages of change for exercise behavior. Am J Health Promot 12, 68-74.

17. Food Standards Agency (2008) The eatwell plate. http://www. eatwell.gov.uk/healthydiet/eatwellplate (accessed July 2008).

18. Baker A, Bakhshi S, Suruilal-Harry A et al. (2005) The efficacy of the theory of planned behaviour in predicting dietary behaviour for different foods in different groups. Psychol Health 10, Suppl. 1, 19.

19. Roe L, Strong C, Whiteside C et al. (1994) Dietary intervention in primary care: validity of the DINE method for assessment. Fam Pract 11, 375-381.
20. Cohen J (1992) Quantitative methods in psychology: a power primer - group of 3. Psychol Bull 1, 155-159.

21. Campbell MK, Elbourne DR \& Altman DG (2004) CONSORT statement: extension to cluster randomised trials. BMJ 328, 702-708.

22. Pinheiro JC \& Bates DM (2000) Mixed-Effects Models in $S$ and S-PLUS. New York: Springer Verlag.

23. Rigby RA \& Stasinopoulos DM (2005) Generalized additive models for location, scale and shape (with discussion). Appl Stat 54, 507-554.

24. Bingham SA, Gill C, Welch A et al. (1994) Comparisons of dietary assessment methods in nutritional epidemiology: weighed records v. $24 \mathrm{~h}$ recalls, food frequency questionnaires and estimated diet records. Br J Nutr 72, 619-643.

25. Ritterband LM, Gonder-Frederick LA \& Cox DJ (2003) Internet interventions: in review, in use, and into the future. Prof Psychol Res Pr 34, 527-534.

26. Heimendinger J, O'Neill C, Marcus AC et al. (2005) Multiple tailored messages are effective in increasing fruit and vegetable consumption among callers to the Cancer Information Service. J Health Commun 10, Suppl. 1, 65-82.

27. Giroti A, Smith MA, Walker L et al. (1995) Increasing fruit and vegetable consumption in second and third grade students. J Am Diet Assoc 95, 9 Suppl. 1, 37.

28. Mangunkusumo R, Brug J, de Koning $\mathrm{H}$ et al. (2007) School-based Internet-tailored fruit and vegetable education combined with brief counselling increases children's awareness of intake levels. Public Health Nutr 10, 273-279.

29. Haerens L, De Bourdeaudhuij I, Maes L et al. (2007) The effects of a middle-school healthy eating intervention on adolescents' fat and fruit intake and soft drink consumption. Public Health Nutr 10, 443-449.

30. Campbell M, DeVellis BM, Strecher VJ et al. (1994) Improving dietary behavior: the effectiveness of tailored messages in primary health settings. Am J Public Health 84, 783-787.

31. Campbell MK, Tessaro I, DeVellis B et al. (2002) Effects of a tailored health promotion program for female bluecollar workers: health works for women. Prev Med 34, 313-323.

32. Adamson AJ, Curtis PJ, Loughridge J et al. (2000) A familybased intervention to increase consumption of starchy foods. Nutr Food Sci 30, 19-23.

33. Comrie F, Cochrane N, Aucott L et al. (2006) Design and implementation of an Internet dietary assessment method for use in a student population. Poster presented at 6 th International Conference on Dietary Assessment Methods, Copenhagen, 27-29 April 2006.

34. Hyskey S \& Troop NA (2002) Online longitudinal survey research: viability and participation. In Psychology and the Internet (special issue). Soc Sci Comput Rev Arch 20, 250-259.

35. Oenema A, Brug J \& Lechner L (2001) Web-based tailored nutrition education: results of a randomised controlled trial. Health Educ Res 16, 647-660.

36. Mangunkusumo RT, Brug J, Duisterhout JS et al. (2007) Feasibility, acceptability, and quality of Internet-administered adolescent health promotion in a preventive-care setting. Health Education Res 22, 1-13.

37. Fotheringham MJ, Owies D, Leslie E et al. (2000) Interactive health communication in preventive medicine. Internetbased strategies in teaching and research. Am J Prev Med 19, 113-120. 